\title{
Los indicadores ambientales como herramientas de la economía
}

Estela Monica Lopez Sardi ${ }^{1}$ y Maricel Patricia Cattaneo ${ }^{1}$

\section{Resumen}

Desde la primera mitad del siglo XX, las distintas corrientes del pensamiento económico han buscado la forma más efectiva de incorporar las externalidades ambientales a las cuentas de la economía. Las metodologías van desde la valoración monetaria del ambiente propuesta por la economía ambiental de la escuela neoclásica, hasta la expresión de la economía en términos de energía propuesta por la economía ecológica. El desarrollo de las naciones y el bienestar de las poblaciones se han estimado tradicionalmente por indicadores económicos como el Producto Bruto Interno. Estos indicadores ponderan las variables involucradas en su cálculo en términos monetarios. Sin embargo, estos índices no permiten evaluar el escenario económico en términos de sostenibilidad. Los indicadores de sostenibilidad, que expresan las externalidades ambientales en unidades físicas, no monetarias, deberían cada vez más, ser incorporados al campo de los negocios, constituyéndose en las nuevas herramientas de la economía sostenible. El presente artículo propone un recorrido a través de los mecanismos económicos destinados a incorporar el ambiente como objeto de estudio. Así mismo se destaca la importancia de incorporar los indicadores ambientales al conjunto de las herramientas clásicas de la economía.

Palabras Clave: indicadores ambientales, sostenibilidad, economía, ambiente 


\begin{abstract}
Since the first half of the twentieth century, the different schools of economic thought have sought the most effective way to incorporate environmental externalities to economic accounts. Methodologies ranging from the environmental monetary valuation proposed by the environmental economics of the neoclassical school, up to the expression of the economy in terms of energy proposed by the ecological economics. Nation development and population well-being have been traditionally estimated by economic indicators such as GDP. These indicators weight in monetary terms all the variables involved in the calculation. However, these rates do not allow assessing the economic outlook in terms of sustainability. Sustainability indicators, which reflect environmental externalities in physical and not monetary units, should increasingly be incorporated into the business field, becoming the new tools of sustainable economy. This paper proposes a revision of economic mechanisms addressed to incorporate the environment as a study object. The importance of incorporating environmental indicators to all the classic economics tools is also highlighted.
\end{abstract}

Keywords: environmental indicators, sustainability, economics, environment. 


\section{Introducción}

La actividad económica mundial ha superado la resiliencia natural de los recursos ambientales. Los economistas han considerado históricamente a los bienes naturales como bienes no económicos, dado que se los considera abundantes y en algunos casos, no tienen dueño ni precio. Estos bienes naturales, como el suelo, el agua, el aire, y los recursos no renovables, son de uso imprescindible en los procesos productivos. La degradación que sufren a raíz de la actividad antrópica, provocando agotamiento y contaminación, los transforma en bienes cada vez más escasos, algunos de ellos con horizontes de agotamiento previsibles. (Man $\mathrm{Yu}$ Chang, 2005, p.175).

Se observa una tendencia creciente en los sectores empresarios, de implementar en sus procesos productivos sistemas de gestión ambiental. Los motivos que pueden impulsar a una empresa a tomar este tipo de medidas pueden ser variados. La causa más frecuente de este tipo de decisión radica en la necesidad de adecuar sus actividades a los requisitos legales y normativa vigente. Otro factor determinante para estas acciones, reside en la mirada del consumidor. El público en general se muestra cada vez más interesado en llevar un estilo de vida amigable con el ambiente y socialmente responsable. Un consumidor informado demostrará preferencia por aquellos productos que le permitan minimizar su impacto ambiental a través de sus hábitos de consumo. Así, las acciones de gestión ambiental y de responsabilidad social empresaria representan una ventaja competitiva a la hora de colocar en el mercado los bienes o servicios producidos. (Abarca y Sepúlveda, 2001, p.1).

Distintas escuelas del pensamiento en economía, han comenzado a valorar los bienes ambientales como bienes económicos en función de su escasez presente o futura. El medio ambiente, tradicionalmente considerado externo al mercado, puede internalizarse mediante variadas herramientas metodológicas. Uno de los aspectos fundamentales de este problema, es la determinación del mecanismo para dar valor a bienes considerados invaluables.

Una metodología para establecer las incidencias de los procesos productivos sobre los activos ambientales es la utilización de indicadores biofísicos de sostenibilidad, que expresan sus medidas en unidades físicas no monetarias, tales como hectáreas, m3, Joules, toneladas u otras. Entre los más difundidos podemos citar la huella ecológica, la huella de carbono, la huella hídrica y el agua virtual. La huella de carbono de los bienes y servicios ya ha captado el interés en el campo de los negocios y en ciertos casos, es considerada un factor de riesgo de inversión. Un gran número de empresas multinacionales está implantando políticas estrictas de control de emisiones, huella de carbono y huella hídrica. (Civit, 2011, párr. 6 a 8). En un futuro no muy lejano, la certificación medioambiental será indispensable para el posicionamiento empresario en el escenario de los negocios internacionales. 
El presente artículo propone un recorrido a través de los mecanismos económicos destinados a incorporar el ambiente como objeto de estudio. Así mismo se destaca la pertinencia de incorporar los indicadores ambientales al conjunto de las herramientas clásicas de la economía.

\section{Incorporación del ambiente a la economía}

La escuela económica neoclásica o keynesiana al incorporar el medio ambiente como objeto de estudio, dio lugar al nacimiento de la economía ambiental. La economía neoclásica basa su análisis en los precios del mercado, con una concepción crematística, donde la realidad económica funciona como un móvil perpetuo, de ciclo cerrado, en el que las empresas venden bienes y servicios a los consumidores, y con el dinero obtenido de las transacciones de mercado remuneran los factores de producción tales como tierra, trabajo y capital, con los cuales realimentar el ciclo. (Martínez Alier, 1998, p.12).

La economía ambiental, que adjudica a los bienes un precio en función de su escasez, comienza a adjudicar valor al medio ambiente. Para lograr esto se desarrollan estrategias de internalización de las externalidades. Todos los efectos involuntarios de una determinada actividad económica sobre el bienestar general de la sociedad, el ambiente o las demás empresas se denominan externalidades. Si estos efectos son beneficiosos, se las considera externalidades positivas y no causan preocupación. Si los efectos son perjudiciales, se las considera externalidades negativas, costos privados que generalmente son pagados por la sociedad en general. La internalización de las externalidades consiste, en el caso de verse afectados los factores ambientales, en adjudicar un valor monetario al medio ambiente, el que pasa a tener un precio o un derecho de propiedad. (Man Yu Chang, 2005, p.175).

Arthur Cecil Pigou, en The economics of welfare, (1920), fue el primero en definir el concepto de internalización de las externalidades. Reconoce que, salvo bajo la situación ideal de competencia perfecta, existen muchas fallas en el mercado. Estas fallas implican que la maximización del bienestar privado no coincida con la maximización del bienestar social. En consecuencia propone al Estado como el árbitro encargado de reglamentar y disciplinar estos efectos externos. Las medidas a adoptar para lograr este objetivo, actualmente pueden ir desde el establecimiento mediante normativa de límites tolerables de vertido de los distintos contaminantes hasta el cobro de impuestos sobre la contaminación.

Otra escuela de pensamiento, dentro de la economía ambiental, es la de Ronald Coase, inglés que se integró a la Escuela de Chicago, (1960), quien preconiza que las externalidades deben ser resueltas entre las partes privadas involucradas sin intervención del estado. Si el recurso pertenece al contaminado, el contaminador 
debe pagar por contaminar; si el recurso pertenece al contaminador, el contaminado es el que debe pagar para compensar las pérdidas económicas del contaminador debidas al no uso del recurso. (Man Yu Chang, 2005, p.180).

Este tipo de acuerdo entre las partes involucradas es conocido como "negociación coasiana". Parafraseando a Martínez Alier (1998), p. 29; se puede ejemplificar como sigue: La empresa A, pongamos por caso una minera o una fábrica de pasta de papel, contamina el agua de un río X. Aguas abajo se encuentra la empresa B, que podría ser un emprendimiento agrícola que usa el agua del río $\mathrm{X}$ para regadío. La actividad de la empresa A (el contaminador) perjudica a la empresa B (el contaminado). Si la empresa A tiene un derecho implícito sobre el recurso (en este caso el río X) será la empresa B la que deba asumir los costos de tratamiento del agua para poder utilizarla, caso contrario, si los derechos sobre el recurso los tiene la empresa B, será la empresa A quien deba asumir los costos de tratamiento del agua para que pueda ser usada por la empresa B.

Las herramientas de la economía ambiental son las más aplicadas en la actualidad para la toma de decisiones de negocios que impliquen factores ambientales. Este tipo de negociación puede llegar a funcionar cuando se trata de externalidades mutuas entre empresas, o entre empresas y consumidores, siempre y cuando lleguen a un acuerdo sobre el valor monetario de las externalidades implicadas en el problema. Sin embargo, en los casos de externalidades que afectan bienes invaluables como la biodiversidad, el futuro de los recursos o el bienestar de las generaciones presentes o futuras, suele resultar de difícil aplicación.

Entre los años 1970 y 1980, de la mano de Nicholas Georgescu - Roegen ve la luz una nueva corriente del pensamiento conocida como economía ecológica. Esta ciencia transdiciplinar valoriza el ambiente desde la mirada de la naturaleza: de los ciclos biológicos, geológicos, y el intercambio de energía.

Georgescu - Roegen, en The entropy law and the economics process, (1971), pone de manifiesto que el planeta Tierra es un sistema abierto a la entrada de energía solar, utilizada en los distintos procesos, tanto biológicos como industriales productivos (energía libre utilizable), para luego transformarse en calor disipado (energía restringida inutilizable). Algo similar ocurre con el uso de los recursos materiales, los cuales, según el principio de conservación la materia no se destruyen, pero la actividad humana los transforma finalmente en residuos, los cuales pueden ser sólo parcialmente recuperados mediante el reciclaje. Todos los procesos están sujetos a las leyes de la termodinámica y los procesos económicos no constituyen una excepción.

Se propone un modelo del proceso productivo como si se analizara a un organismo vivo, con entradas de materia, energía e información provenientes del ambiente y los recursos. Las salidas desde el proceso hacia el ambiente consisten en residuos y energía disipada. Este proceso, aplicado al análisis de individuos, procesos o comunidades, es conocido como metabolismo social. (Rovira, 2011, párr.4). 
Esta escuela propone mantener un equilibrio entre la población, los recursos finitos y el ambiente, para lograr una supervivencia de nuestras culturas que cumpla con el principio de sostenibilidad. Esta corriente del pensamiento constituye un contrapunto a la economía ambiental al contabilizar el patrimonio natural en unos términos que no son necesariamente los del dinero.

El desarrollo sostenible se basa en el concepto de estado estacionario propuesto por Herman Daly, discípulo de Georgescu. Este principio postula una situación ideal de población constante, un conjunto de material físico (artefactos) constante, y un uso mínimo de materiales y energía. El propio Georgescu duda de la posibilidad de alcanzar este estado y propone un escenario de necesario decrecimiento como el único posible para asegurar el bienestar de las generaciones futuras.

\section{Indicadores económicos de bienestar}

El Producto Bruto Interno (PBI), es una medida macroeconómica que expresa el valor total de la producción de bienes y servicios de un país durante un ejercicio fiscal (generalmente un año). Es la base del cálculo del ingreso per cápita y muchas veces es utilizado como medida del bienestar material de una sociedad. Este indicador, ampliamente utilizado, ha recibido muchas críticas, como las del Profesor Joseph E. Stiglitz (premio Nobel de Economía), quien manifestó que el PBI “...sólo compensa a los gobiernos que aumentan la producción material. [...] no mide adecuadamente los cambios que afectan al bienestar, ni permite comparar correctamente el bienestar de diferentes países[...] no toma en cuenta la degradación del medio ambiente ni la desaparición de los recursos naturales a la hora de cuantificar el crecimiento..." (Stiglitz, 2008, párr. 1 a 11).

Para poder contabilizar las externalidades negativas, incluir los efectos de las actividades productivas sobre el ambiente y establecer una vinculación con el bienestar social se han propuesto otro tipo de indicadores. Entre ellos figura el PBI verde, obtenido al restar del PBI el valor de los recursos naturales deteriorados por la actividad económica del ejercicio fiscal. En 2004 el Partido Comunista Chino anunció su intención de reemplazar el cálculo del PBI por el PBI verde. Esta iniciativa fue rápidamente abandonada ya que las reducciones en el PBI tras la corrección terminaron arrojando cifras políticamente inaceptables. (Carrillo, 2007, párr. 1 a 4).

El Programa de las Naciones Unidas para el Desarrollo (PNUD) propone el cálculo del Índice de Desarrollo Humano (IDH), basado en la salud (a partir de esperanza de vida al nacer), la educación (en base a la tasa de alfabetización y nivel de estudios alcanzado) y en el nivel de vida (calculado a partir del PBI per cápita). (Pampillón, 2009, párr.1). 
El índice de bienestar económico sostenible (IBES), es una visión más amplia del PBI, que considera el gasto de los consumidores y la utilidad aportada por el trabajo doméstico y descuenta las externalidades asociadas al uso de los recursos naturales y la polución ambiental. Del IBES derivan el índice de progreso real (IPR) y el índice de progreso genuino (IPG), originados en las ideas presentadas por los economistas Nordhaus, W. y Tobin, J. en su Measure for Economic Welfare, (1972). Las críticas a estos índices radican en que los países de muy bajo desarrollo económico podrían arrojar valores altos del índice debido a sus muy bajos niveles de polución.

Los descriptos constituyen una pequeña muestra del universo de los indicadores económicos utilizados para valorar el bienestar de una población. Al ser incluidos en el cálculo de estos índices, los impactos ambientales derivados de la actividad humana se consideran externalidades que se traducen en un valor monetario.

\section{Indicadores ambientales}

A diferencia de los anteriores, los indicadores biofísicos de sostenibilidad ambiental, tales como las huellas (ecológica, de carbono e hídrica) permiten valorizar el impacto ambiental de las distintas actividades antrópicas en términos de unidades físicas. Un indicador ambiental que arroje resultados altos para una región o nación determinada, incidirá, a corto o largo plazo, negativamente en su PBI y en su IDH, al denotar una actividad económica generadora de externalidades negativas de consideración.

Las huellas ambientales de los bienes y servicios producidos en una economía pueden ser calculadas a partir de las cuentas contables de las empresas productoras. Al avanzar el producto en la cadena de valor, la información fluye de una organización a otra, en forma acumulativa, llegando al consumidor con una huella final del producto definida como la suma de todas las huellas necesarias para crear, usar y/o destruir ese producto. El valor global de las huellas de la cadena productiva nacional se obtendrá a partir de la sumatoria de los valores individuales y considerando, cuando esto sea necesario, las magnitudes asociadas a la importación y exportación de productos.

\section{La huella ecológica}

La huella ecológica se define como el área de tierra (o agua) ecológicamente productiva requerida para generar los recursos consumidos y asimilar los residuos generados por una población o comunidad con un estilo de vida específico. Fue definida en 1996 por William Rees y Mathis Wackernagel (Universidad de la 
Columbia Británica). Se la puede calcular a varias escalas, desde el nivel personal u hogareño hasta la huella ecológica de un país e incluso a nivel planetario.

La biocapacidad es la capacidad de un área específica para generar un abastecimiento regular de recursos renovables y absorber los desechos resultantes de su consumo. (Rosso, 2010, párr. 2).

El consumo de los distintos bienes y servicios se convierte en la superficie biológica productiva asociada mediante índices de productividad. Estos índices se refieren a seis categorías de áreas diferentes y se expresan en gHa (hectáreas globales, unidad común que unifica las demandas de bio - recursos). Las seis categorías son:

Área de producción de cultivos y vegetales.

Área dedicada al pastoreo de ganado.

Área de explotación de bosques.

Área acuática para la producción de pescado y marisco.

Áreas urbanizadas u ocupadas por infraestructuras.

Área de absorción de dióxido de carbono, pulmones verdes destinados a absorber las emisiones provenientes del uso de combustibles fósiles.

La capacidad de carga de un territorio representa la superficie biológicamente disponible, teniendo en cuenta la productividad del terreno y reservando un $12 \%$ de la misma para el mantenimiento de la biodiversidad. La capacidad de carga planetaria está estimada en 1,75 gHa / habitante. La huella ecológica media mundial, calculada en base a los niveles de consumo del año 2005, es de 2,7 gHa / habitante, lo que indicaría una sobreexplotación del $60 \%$ por encima de la capacidad de carga del planeta. Estamos consumiendo más recursos y generando más residuos de los que el planeta puede generar y admitir. (Moreno López, 2005, párr. 1 a 3).

La biocapacidad de la Argentina es de 7,5 gHa/ habitante. La huella ecológica nacional se ubica en 2,6 gHa/ habitante; tan sólo el $35 \%$ de la biocapacidad del país. En función de estos valores, es posible afirmar que Argentina "financia ecológicamente" a otros países que se encuentran en déficit. (Rosso, 2010, párr. 7).

\section{Agua virtual y huella hídrica}

El concepto de agua virtual (AV), desarrollado en 1993 por el Profesor John Anthony Allan, de la Universidad de Londres, se define como la cantidad de agua requerida para producción de los bienes agrícolas o industriales. En 2002, el Profesor Arjen Hoekstra, de UNESCO-IHE (Institute for Water Education), introdujo el más amplio concepto de huella hídrica $(\mathrm{HH})$ como un indicador para evaluar el uso del agua. La huella hídrica establece el volumen total de agua consumida, evaporada o contaminada durante la producción de bienes y servicios por parte de una empresa $\mathrm{u}$ organización, o durante las actividades desarrolladas por un individuo, familia, 
comunidad o nación. Incluye la producción, transformación, comercialización y consumo de los productos. El cálculo se realiza en $\mathrm{m} 3 / \mathrm{kg}$ para la producción de bienes y en m3/año para el consumo por parte de individuos o comunidades. Se debe tener en cuenta que la huella hídrica de una región o estado se realiza teniendo en cuenta en el balance la importación y la exportación de agua virtual.

El país con mayor $\mathrm{HH}$ a nivel mundial es EEUU con $2.483 \mathrm{~m} 3$ anuales per cápita, y el de menor $\mathrm{HH}$ es China con $700 \mathrm{~m} 3$ anuales per cápita. Este balance, surgido de la diferencia $\mathrm{HH}$ regional $=$ Consumo interno $+\mathrm{AV}$ importada $-\mathrm{AV}$ exportada, supone un deterioro de los recursos de los países exportadores y un importante ahorro de agua en los países importadores. Se estima que el 15\% del agua consumida en el mundo está destinada a ser exportada como agua virtual. Un $67 \%$ del agua virtual exportada por el conjunto de naciones, está relacionado con el comercio internacional de cultivos, $23 \%$ con el comercio de ganado y un $10 \%$ con la producción industrial. La Argentina se encuentra en el cuarto lugar dentro de la lista de países exportadores de AV, superado sólo por E.E.U.U., Canadá y Tailandia mientras que Brasil aparece en el décimo lugar. (Hoekstra y Hung, 2002, pág. 26).

Datos publicados por el INTA en 2010 y corroborados en 2012, indican que, sólo en materia de granos, exportamos 46.000 millones de $\mathrm{m} 3$ de agua e importamos 3.100 millones. Según Enrique Sánchez, especialista del INTA, Alto Valle, Río Negro, (2012), sería interesante “...hacer notar al país comprador la cantidad de agua que ahorra y que puede direccionar a otras necesidades esenciales de su población $[\ldots]$ se debería sacar ventaja del agua virtual que se exporta...".

La tabla 1 nos da una idea del agua virtual comprometida en la producción de una serie de productos agrícolas e industriales.

\begin{tabular}{|l|l|l|l|}
\hline \multicolumn{1}{|c|}{ Producto } & \multicolumn{1}{c|}{ Agua virtual } & \multicolumn{1}{c|}{ Producto } & \multicolumn{1}{c|}{ Agua virtual } \\
\hline Manzanas & $0,4 \mathrm{~m} 3 / \mathrm{kg}$ & Papel & $2 \mathrm{m3} / \mathrm{kg}$ \\
\hline Carne vacuna & $10 \mathrm{a} 40 \mathrm{~m} 3 / \mathrm{kg}$ & Tela de algodón & $8 \mathrm{~m} 3 / \mathrm{kg}$ \\
\hline $8 \mathrm{~m} 3 / \mathrm{kg}$ & $3,92 \mathrm{m3} / \mathrm{kg}$ & Zapatos de cuero & $8 \mathrm{~m} 3 / \mathrm{par}$ \\
\hline Arroz & $3 \mathrm{~m} 3 / \mathrm{kg}$ & Oro & $230 \mathrm{~m} 3 / \mathrm{kg}$ \\
\hline Trigo & $1 \mathrm{~m} 3 / \mathrm{kg}$ & Microchips & $16 \mathrm{~m} 3 / \mathrm{kg}$ \\
\hline
\end{tabular}

Tabla 1. Agua virtual de productos.

\section{Huella de carbono}

La huella de carbono es una medida del impacto que las actividades humanas tienen en el ambiente en términos de emisión de gases de efecto invernadero (GEI), calculada en toneladas anuales de dióxido de carbono, lo que permite que organizaciones, productos o personas puedan conocer su influencia en el cambio climático. Este 
indicador deriva de la huella ecológica definida por Rees y Wackernagel.

Puede ser calculada para distintos niveles: huella de carbono personal, de productos, de procesos, empresas, eventos o naciones.

El método consiste en traducir todos los impactos ambientales, tanto consumos como desechos, en la cantidad de dióxido de carbono equivalente emitido. (Instituto Huella de Carbono, 2010, párr.1).

La herramienta para realizar esta conversión son los factores de emisión calculados para cada una de las instancias evaluadas, y la unidad de expresión es toneladas anuales de dióxido de carbono (en adelante, TACO2). La principal dificultad del método estriba en seleccionar para el cálculo un conjunto de factores de emisión consistentes. Es preciso tener en cuenta que para una misma actividad los factores de emisión pueden ser distintos según la región y el método de producción.

El cálculo de la huella de carbono personal se realiza en base al análisis de los datos suministrados por las personas individuales respecto de su estilo de vida. El cuestionario utilizado para su evaluación, está estructurado en distintas secciones, que abarcan los puntos significativos de emisión personal, como ser consumo hogareño de energía, transporte, alimentación, consumo de bienes y servicios y generación de residuos. Cada uno de estos consumos tiene su propia huella de carbono que es heredada por el usuario. Los consumos familiares se prorratean según el número de habitantes en el hogar.

El promedio mundial de la huella de carbono personal es de $4 \mathrm{TACO} 2$ mientras que el objetivo es alcanzar un valor de $2 \mathrm{TACO} 2$ por cada habitante del planeta. (Carbonfootprint, 2013, pág. 8).

En 2008, la Secretaria de Ambiente y Desarrollo Sustentable de la Nación obtuvo, mediante datos del Instituto Nacional de Estadísticas y Censo (INDEC), una huella de carbono personal para la Argentina de 5,71 TACO2.

El trabajo desarrollado durante el período 2011 - 2012 por el Centro de Investigación en Ingeniería Sustentable (CIIS) de la Facultad de Ingeniería de la Universidad de Palermo, arroja un valor promedio de 5,95 TACO2 por habitante en la región de la Ciudad de Buenos Aires (CABA) y Gran Buenos Aires (GBA), distribuidos como se muestra en la Tabla 2:

\begin{tabular}{|l|l|}
\hline \multicolumn{1}{|c|}{ Actividad } & TACO2 / PERSONA \\
\hline Traslados diarios & 0,5 \\
\hline $\begin{array}{l}\text { Traslados de larga distancia } \\
\text { (negocios o turismo) }\end{array}$ & 1,75 \\
\hline Uso de energía en el hogar & 1,14 \\
\hline Alimentación & 1,63 \\
\hline Residuos & 0,02 \\
\hline Consumo de bienes y servicios & 0,91 \\
\hline
\end{tabular}

Tabla 2. Huella de carbono personal para CABA y GBA 
Las calculadoras de huella de carbono personal generalmente se acompañan de una serie de recomendaciones ambientales que permitirán al interesado adoptar cambios en su estilo de vida para lograr una disminución de emisiones.

Respecto de la huella de carbono de organizaciones o productos, se pueden calcular de acuerdo a los diferentes estándares internacionales.

Para determinar la huella de carbono de los productos, vinculada al análisis de ciclo de vida (LCA) de los mismos, los principales estándares están establecidos por las Normas PAS 2050 e ISO 14067.

La huella de carbono de una organización abarca todos los sectores y actividades que tienen lugar dentro de la estructura y los principales estándares son los GHG Protocol y las Normas ISO $14064-1$.

Una de las metodologías más utilizadas para el cálculo de la huella de carbono de una organización es la MC3, desarrollada por Domenech. Este método incluye en el cálculo tanto las emisiones directas, generadas por la empresa u organización analizada en sus distintos sectores de gestión, como las emisiones indirectas que derivan de la adquisición de bienes y servicios a terceros. El método permite también el cálculo de la huella de carbono de un producto a lo largo de la cadena de suministro, basado en la huella de carbono de las empresas que participan de esa cadena.

El MC3, aplicado originalmente por la Autoridad Portuaria de Guijón, fue testeado por universidades españolas y es actualmente utilizado por gran número de empresas de la Comunidad Económica Europea. La información necesaria para calcular la huella de carbono corporativa según este método, proviene de los documentos contables de la empresa (balance, cuentas de pérdidas y ganancias) sumada a la suministrada por otros sectores, como por ejemplo, generación de residuos o superficie destinada a los distintos usos. La determinación de la huella se realiza mediante una hoja de cálculo donde se consigna toda la información por sector. (Carballo Penela, García Negro, Domenech, 2009, pág. 1 a 16).

\section{Conclusión}

El desarrollo sostenible es un tema central de la agenda mundial del siglo XXI. El concepto es utilizado por la política, la sociología, la ecología, la economía y la ingeniería, entre otras ciencias. Se lo discute en foros internacionales y se intenta llevarlo a la práctica en experiencias con mayor o menor suceso. Una de las mayores dificultades para encontrar un punto de entendimiento general estriba en que cada ciencia maneja su propio lenguaje y herramientas. Es prioritario desarrollar un paradigma nuevo, transdisciplinar y ajustado a los mecanismos de la naturaleza, que permita realmente satisfacer las necesidades 
de las generaciones presentes sin comprometer el bienestar de las generaciones futuras. La incorporación definitiva a la economía de indicadores ambientales capaces de expresar las externalidades ambientales en términos no monetarios, puede ser un primer paso en la búsqueda de ese lenguaje común. 


\section{Bibliografía}

Abarca, R.; Sepúlveda, S. (2001). Eco - etiquetado. Un instrumento para diferenciar productos e incentivar la competitividad. Cuaderno Técnico $\mathrm{N}^{\circ} 17$. Obtenido el 14 de junio de 2013 en http://www.iica.int/Esp/regiones/central/ honduras/Publicaciones\%20IICA/Competitividad\%20y\%20Agronegocios/ Ecoetiquetado_Un_instrumento_para_diferenciar_productos_e_incentivar_la_ competitividad.pdf

Carballo Penela, A.; García Negro, M.; Domenech Quesada, J. (2009). El MC3 una alternativa metodologica para estimar la huella corporativa del carbono (HCC). Revista de desarrollo local sostenible. DELOS. Obtenido el 15 de junio de 2013 en www.eumed.net/rev/delos/05

Carbonfootprint. (s.f.). Calculadora de huella de carbono. Obtenido el 15 de junio de 2013 en http://calculator.carbonfootprint.com/calculator.aspx?lang=es\&tab=8

Carrillo, J. (2007). El PBI verde sonroja a China. Economy Weblog. Obtenido el 14 de junio de 2013 en http://economy.blogs.ie.edu/archives/2007/07/el_pib_ verde_so.php

Chang, M.Y. (2005). La economía ambiental. Obtenido el 7 de junio de 2013 de http://meme.phpwebhosting.com/ migracion/rimd/coleccion_america_latina/ sustentabilidad/Sustentabilidad9.pdf

Civit, B. (2011). Huella de carbono, ecológica y huella hídrica. Correveidile. La voz de Chacras. Obtenido el 10 de junio de 2013 en http://www.correveidile.com. ar/2011/03/02/huella-ecologica-huella-de-carbono-y-huella-hidrica/

Georgescu - Roegen, N. (1971). The entropy law and the economic process. Harvard University Press: Cambridge, Massachusetts.

Hoekstra, A.Y.; Hung,P.Q. (2002). Virtual water trade. A quantification of virtual waters flows between nations in relation tointernational crop trade. Obtenido el 11 de junio de 2013 de http://www.waterfootprint.org/Reports/Report11.pdf

Instituto Huella de Carbono S. L. (2010). Cálculo de la huella de carbono. Metodología MC3. Obtenido el 15 de junio de 2013 en http://www. institutohuelladecarbono.com/gestion-y-politicas-de-cambio-climatico/-calculode-la-huella-de-carbono-metodologia-mc3.html

Madrid, C. (2007). Hidratar el metabolismo socioeconómico: agua virtual y el metabolismo hídrico. Obtenido el 7 de junio de 2013 de http://ddd.uab.cat/pub/ 
trerecpro/2007/hdl_2072_13466/TR+Cristina+Madrid.pdf

Martínez Alier, J. (1998). Curso de Economía Ecológica. Serie Textos Básicos para la Formación Ambiental $N^{\circ} 1$. Red de Información Ambiental. Programa de las Naciones Unidas para el Medio Ambiente. Oficina Regional para América Latina y el Caribe.

Moreno López, R. (2005). La huella ecológica. Obtenido el 14 de junio de 2013 en http://habitat.aq.upm.es/boletin/n32/armor.html

Pampillón, R. (2009) ¿Qué es el indice de desarrollo humano? Economy Weblog. Obtenido el 14 de junio de 2013 en http://economy.blogs.ie.edu/ archives/2009/10/\%C2\%BFque-es-el-indice-de-desarrollo-humano-idh.php

Pérez Rincón, M.A. (2009). Indicadores biofisicos de sustentabilidad. Sesión 4. Obtenido el 7 de junio de 2013 de HTTP://WWW.PUMA.UNAM.MX/DOC/ TALLER03-INDICADORES-BIOFISICOS.PDF

Pigou, A. C. (1932). The economics of welfare. 4ta edición. Mcmillan and Co. Londres.

Quiroga, A. (2012). Huella hídrica: eficiencia en el uso del agua. Obtenido el 15 de marzo de 2013 de http://intainforma.inta.gov.ar/?p=12694

Rocha,L. (2009). También hay agua virtual. Obtenido el 6 de junio de 2013 de http://blogs.lanacion.com.ar/ecologico/el-ambiente-en-general/tambien-hayagua-virtual/

Rosso, A. (2010) ¿Cuán grandes son nuestros pies? Programa Huella Ecológica y Biocapacidad Argentina. INTI. Obtenido el 15 de junio de 2013 en http://www. inti.gob.ar/sabercomo/sc95/inti9.php

Rovira, P.(2011). Metabolismo social.¿Qué es? Obtenido el 6 de junio de 2013 en http://batzolades.wordpress.com/2011/03/23/metabolismo-social-\%C2\%BFque-es/

Sánchez, E. (2012). La huella hídrica para agregar valor. Obtenido el 15 de marzo de 2013 de http://intainforma.inta.gov.ar/?p=3642

Stiglitz, J. E. (2008). Cuestiona Stiglitz efectividad del PBI para medir el crecimiento de los paises. Diario La Jornada, México, Edición 01/09/2008 obtenido el 14 de junio de 2013 en http://www.jornada.unam.mx/2008/01/09/ index.php?section=economia 